\title{
Relationship between cholesterol synthesis/absorption marker and vascular function in healthy subjects
}

\author{
Yutaka Ishibashi $^{1)}$, Kenji Karino ${ }^{2)}$, Shozo Yano ${ }^{2)}$, Tsunetaka Kijima ${ }^{1)}$, Nobuyuki Takahashi ${ }^{1)}$, \\ Shingo Yamagata ${ }^{1)}$, Kazutaka Kurokouchi ${ }^{1}$, Haruhiko Nagami ${ }^{1)}$, Satoshi Honda ${ }^{1)}$ and Yoshihiro Nosou ${ }^{1)}$
}

\begin{abstract}
:
Recently, inhibition of absorbed cholesterol has been reported to reduce the recurrence of vascular events in patients with acute coronary syndrome. However, the effect of absorbed cholesterol on atherosclerosis in healthy subjects remains unclear. In the present study, we investigated the relationship between cholesterol synthesis and absorption biomarkers, and vascular elasticity or morphological changes in healthy subjects. Methods: Among 580 subjects who underwent a complete medical checkup between 2012 and 2015, 256 healthy subjects (male/female: 161/95; mean age, $55 \pm 10$ years) who did not receive any medication were included in the present study. We measured blood pressure, heart rate, blood glucose level, lipid parameters, lathosterol level (cholesterol synthesis marker), and campesterol level (cholesterol absorption marker). We then analyzed the relationship between these measured values and vascular elasticity (cardio-ankle vascular index [CAVI]) or carotid arterial plaque score (CAPS). Results: The CAVI and CAPS significantly correlated with the campesterol-tolathosterol ratio (CAVI; $r=-0.20, \mathrm{p}=0.015$; CAPS; $r=-0.16, \mathrm{p}=0.023$ ). Multivariate analysis of conventional atherosclerotic factors and campesterol-to-lathosterol ratio for CAVI and CAPS as the objective variables showed that age, systolic blood pressure, low-density lipoprotein-to-high-density lipoprotein ratio, and campesterol-to-lathosterol ratio were the significant determining factors for CAVI, and age and campesterol-to-lathosterol ratio were the determining factors for CAPS. Discussion: We found that absorbed cholesterol is closely related to vascular elasticity and morphological changes, and that a higher balance of absorption could induce favorable effects on atherosclerosis in healthy subjects. These findings might propose new nutritional guidance for healthy subjects.
\end{abstract}

Key words:

Campesterol, Lathosterol, Arterial stiffness, Arterial plaque

\section{Introduction}

Cholesterol as an atherosclerotic factor is supplied by synthesis in the liver and absorption from the small intestine. Clinical studies have shown that decreased serum cholesterol level due to synthesis and absorption inhibitors could reduce vascular events ${ }^{1-3}$. Furthermore, the balance of synthesized and absorbed cholesterols could play a role for progression of atherosclerosis ${ }^{4}$. However, study results regarding the relationship between the balance of synthesized and absorbed cholesterols and atherosclerosis are conflicting. Thus, whether absorbed cholesterol could affect atherosclerosis re- mains controversial. Although the confliction could be due to the difference in study participants with or without vascular events, only few studies have been conducted in subjects without vascular events. In the present study, we investigated the relationship among synthesized or absorbed cholesterol markers, vascular elasticity, and vascular morphological changes in healthy subjects.

\section{Methods}

Among 580 subjects who underwent a complete medical checkup between 2012 and 2015, 256 healthy subjects

1) Department of General Medicine, Shimane University Faculty of Medicine

2) Vascular Laboratory, Shimane University Faculty of Medicine

Corresponding author: Yutaka Ishibashi, yuyu@med.shimane-u.ac.jp

Received: September 3, 2017, Accepted: December 25, 2017

Copyright (C) 2018 Japan Society for Vascular Failure 
Table 1. Demographics of the subjects

\begin{tabular}{lcccc}
\hline & $\begin{array}{c}\text { Total } \\
(256)\end{array}$ & $\begin{array}{c}\text { Male } \\
(161)\end{array}$ & $\begin{array}{c}\text { Female } \\
(95)\end{array}$ & $p$ \\
\hline Age & $56 \pm 10$ & $54 \pm 10$ & $56 \pm 9$ & $\mathrm{~ns}$ \\
BMl $\left(\mathrm{kg} / \mathrm{m}^{2}\right)$ & $22.8 \pm 3.2$ & $23.3 \pm 3.0$ & $21.9 \pm 3.2$ & $\mathrm{~ns}$ \\
Abd.circum $(\mathrm{cm})$ & $82 \pm 9$ & $84 \pm 9$ & $78 \pm 9$ & $\mathrm{~ns}$ \\
Smoking $(\mathrm{n})$ & 24 & 23 & 1 & $\mathrm{~ns}$ \\
SBP $(\mathrm{mmHg})$ & $125 \pm 14$ & $128 \pm 13$ & $121 \pm 15$ & $\mathrm{~ns}$ \\
DBP $(\mathrm{mmHg})$ & $76 \pm 10$ & $78 \pm 9$ & $71 \pm 10$ & $\mathrm{~ns}$ \\
TCHO $(\mathrm{mg} / \mathrm{dl})$ & $227 \pm 32$ & $222 \pm 31$ & $235 \pm 33$ & $\mathrm{~ns}$ \\
TG $(\mathrm{mg} / \mathrm{dl})$ & $120 \pm 81$ & $138 \pm 93$ & $91 \pm 44$ & $\mathrm{~ns}$ \\
HDL $(\mathrm{mg} / \mathrm{dl})$ & $65 \pm 17$ & $61 \pm 15$ & $73 \pm 18$ & $\mathrm{~ns}$ \\
LDL $(\mathrm{mg} / \mathrm{dl})$ & $137 \pm 30$ & $135 \pm 29$ & $139 \pm 31$ & $\mathrm{~ns}$ \\
FBS $(\mathrm{mg} / \mathrm{dl})$ & $99 \pm 14$ & $102 \pm 15$ & $95 \pm 8$ & $\mathrm{~ns}$ \\
HbA1c $(\%)$ & $5.5 \pm 0.5$ & $5.5 \pm 0.6$ & $5.5 \pm 0.4$ & $\mathrm{~ns}$ \\
Lathosterol $(\mu \mathrm{g} / \mathrm{ml})$ & $3.05 \pm 1.52$ & $3.42 \pm 1.50$ & $2.45 \pm 1.34$ & $\mathrm{~ns}$ \\
Campesterol $(\mu \mathrm{g} / \mathrm{ml})$ & $6.24 \pm 2.80$ & $6.04 \pm 2.63$ & $6.58 \pm 3.02$ & $\mathrm{~ns}$ \\
\hline
\end{tabular}

Abd. circum: abdominal circumference, SBP: systolic blood pressure, DBP: diastolic blood pressure, TCHO: total cholesterol, TG: triglyceride, HDL: HDL cholesterol, LDL: LDL cholesterol, FBS: fasting blood sugar

(male/female, 161/95; mean age, 55 \pm 10 years) were selected. The levels of blood glucose, lipids (total cholesterol: TCHO, HDL cholesterol: HDL, triglyceride: TG, and LDL cholesterol: LDL), and lathosterol (Latho-S) and campesterol (Camp-S), which are markers of synthesized and absorbed cholesterol, respectively, were measured. The relationship between these measurements and vascular elasticity (cardioankle vascular index: CAVI) or carotid artery plaque score (CAPS) were analyzed. The right and left CAVIs were measured using a CAVIplus VS-1000 system (Fukuda Denshi Ltd, Tokyo, Japan), and the higher CVAI value was used for analysis. Carotid artery ultrasonography was performed using a Vivid S5 system (GE Health Care Japan, Tokyo, Japan) or an Avius ultrasonography imaging system (Hitachi, Tokyo, Japan). The intima-medial thicknesses (IMTs) were measured at $1.5 \mathrm{~cm}$ on the central side of the right and left external-internal carotid artery bifurcations and additional 3 segments toward the peripheral side of the carotid artery at a 1.5-cm interval. The maximal IMT in each segment, with a thickness of $\geq 1.1 \mathrm{~mm}$, was obtained, and summation of the maximal IMTs from 4 segments was defined as CAPS. The lathosterol and campesterol levels were measured using gas chromatography. All the subjects were asked to fast, except water, after $21 \mathrm{pm}$ before the examination. Smoking was also prohibited from a night before.

Statistical analysis of Camp-S/Latho-S ratio and the absolute value of each marker were performed using a JMP ver. 12. Statistical significance was defined as a $\mathrm{p}$ value of $<0.05$. The study was approved by the institutional ethics committee of Shimane University, and written informed consent was obtained from each subject.

\section{Results}

Table 1 shows the demographic characteristics of the subjects. The subjects showed no sex-related differences in age; BMI; systolic and diastolic blood pressures; and levels of TCHO, TG, HDL, LDL, blood glucose, $\mathrm{HbA}_{1 \mathrm{c}}$, lathosterol, or campesterol. Figure 1 shows the distributions of each sterol (panel A) and CAVI (panel B). The lathosterol and campesterol levels varied, with the lathosterol levels showing larger variations. By contrast, the right and left CAVIs showed slight differences with small variations, and the higher values were used for the analysis. Figure 2 shows the relationship between LDL/HDL ratios and absorbed/synthesized cholesterol marker ratio (Camp-S/Latho$\mathrm{S}$ ratio) in all the subjects. Figure 3 shows the relationship between CAVI (panel A) or CAPS (panel B) and Camp-S/ Latho-S ratio. A significant negative correlation was found between LDL/HDL and Camp-S/Latho-S ratio $(r=-0.77, \mathrm{p}=$ 0.0003). CAVI and CAPS also negatively correlated to Camp-S/Latho-S ratio with statistical significance $(r=-0.20$, $\mathrm{p}=0.015$ and $r=-0.16, \mathrm{p}=0.023$, respectively).

The univariate analysis of each factor, using the CAVI and CAPS as objective variables, revealed significant correlations of both CAVI and CAPS to age, systolic blood pressure, diastolic blood pressure, campestrol and Camp-S/ Latho-S ratio (Table 2). Multivariate analysis using the factors with $\mathrm{p}$ values of $<0.1$ as variables revealed that systolic blood pressure, LDL/HDL ratio, and Camp-S/Latho-S ratio were significant determinant factors for CAVI, and that age and Camp-S/Latho-S ratio were significant determinant factors for CAPS (Table 3).

Furthermore, we performed multivariate analysis for CAVI as objective variable using age, Camp-S/Latho-S ratio and CAPS, because we found a significant positive relationship between CAVI and CAPS $(r=0.16, p=0.046)$. In this analysis, age and Camp-S/Latho-S ratio were significant $(\mathrm{p}<0.0001$ and $\mathrm{p}=0.005$, respectively).

\section{Discussion}

In the current cross-sectional study, the absorbed/synthesized cholesterol marker ratio was a significant determinant factor for CAVI and CAPS, indicating that the ratio was equivalent to conventional atherosclerotic risk factors such as age and blood pressure. Our results revealed that balance of absorbed and synthesized cholesterols is closely linked to atherosclerosis progression.

High cholesterol level, especially LDL cholesterol, has been well known to be closely linked to atherosclerosis progression. In humans, cholesterol is supplied by synthesis in liver and absorption from the intestinal tract. Cholesterol synthesis and metabolism pathways in the liver have been well documented, and HMG-CoA reductase inhibitors (statins) are widely used as modulators of the pathway. Statin effects on anti-cerebrovascular and anti-cardiovascular events have already been proven by many large-scale clinical studies ${ }^{1,5)}$. Meanwhile, recently, the effects of inhibition of cholesterol absorption on cardiovascular events was reported. Thus, the inhibitor of Niemann-Pick C1-like protein (NPC1L1) (ezetimibe), which selectively transports choles- 

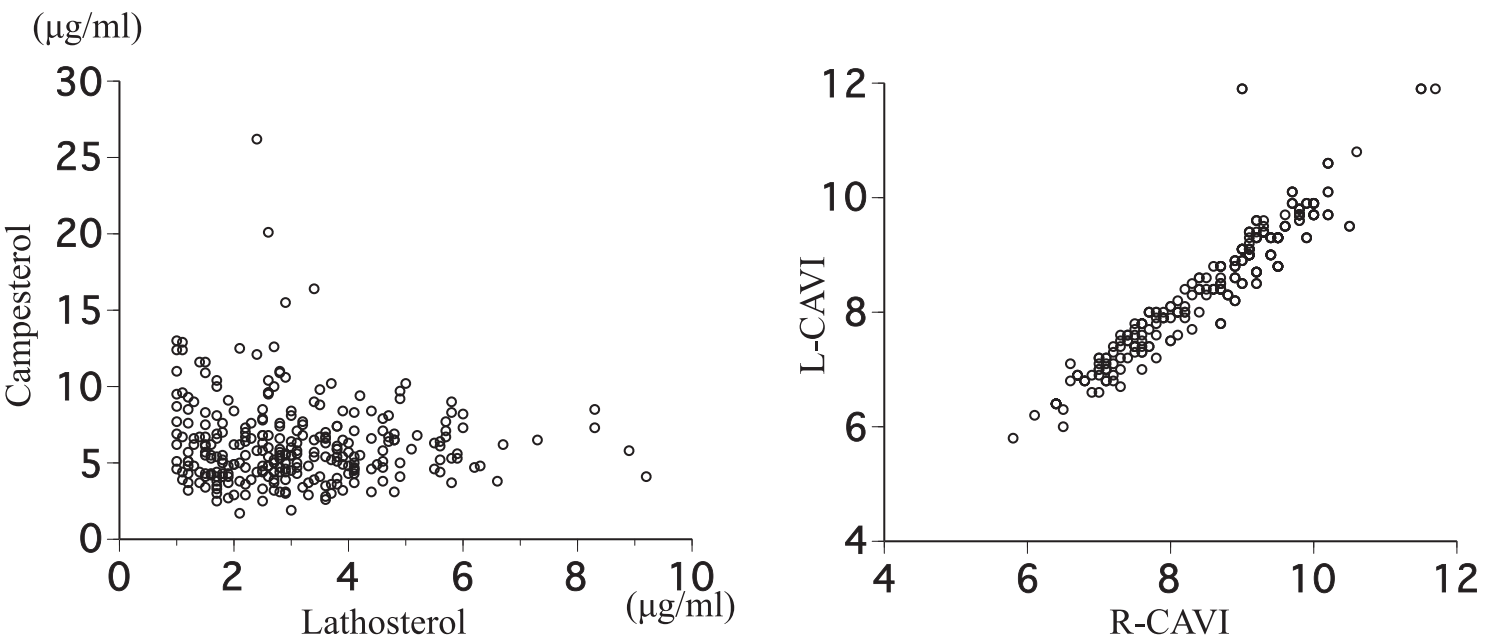

Figure 1. Relationship between absorbed cholesterol (campesterol, vertical axis) and synthesized cholesterol (lathosterol, horizontal axis) in all the subjects (left panel). Relationship between the right (horizontal axis) and left (vertical axis) CAVI measurements (right panel). Both plots indicate variations of the indexes.

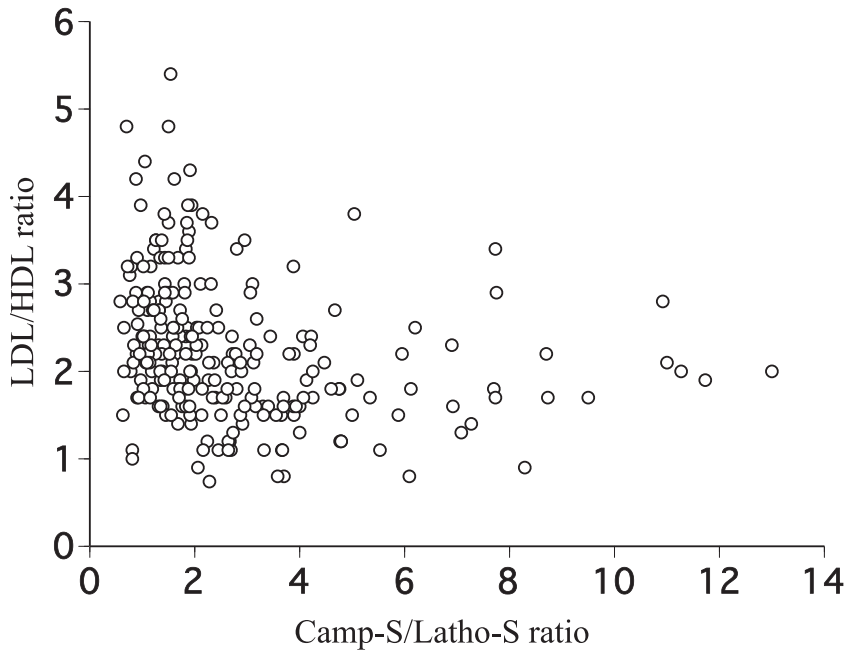

Figure 2. Relationship between LDL/HDL ratio (vertical axis) and absorbed/synthesized cholesterol balance (Camp-S/Latho-S ratio, horizontal axis) in all the subjects. A significant negative correlation was found between the parameters $(r=-0.77, p=0.0003)$.

terol into small intestinal cells ${ }^{6,7)}$, significantly reduced cardiovascular death in 18,144 ACS patients by combined treatment with statin $^{2}$. Recently, Japaridze et al reported that atorvastatin plus ezetimibe therapy significantly reduced vascular events by $11.1 \%$ compared with atorvastatin monotherapy in 323 patients with recently diagnosed acute coronary syndrome $^{3)}$. However, the results of the present study showed that the absorbed cholesterol is not a unique factor of atherosclerosis progression. Thus, the negative correlation between absorbed/synthesized cholesterol ratio and CAVI (vascular elasticity) or CAPS (morphological changes in artery) suggests that higher balance of absorbed blood cholesterol might reduce the atherosclerosis score in healthy subjects.

Fassbennder et al. ${ }^{8)}$ reported in 2008 that the coronary artery disease risk in 1242 healthy subjects with higher ab- sorbed/synthesized cholesterol ratio was reduced at ages $\geq 65$ years. Furthermore, Gylling et al. ${ }^{9}$ measured CAVI in 92 healthy subjects, and Ras et al. ${ }^{10)}$ measured pulse-wave velocity in 240 patients with dyslipidemia and reported that the increased absorbed cholesterol improved vascular elasticity. By contrast, Buchwald et al. ${ }^{11)}$ reported in 1990 that partial resection of the small intestine in 838 patients who survived a first myocardial infarction reduced cardiovascular events (coronary disease death and nonfatal myocardial infarction) by $35 \%$ (ROSCH study). Furthermore, Strandberg et al. ${ }^{12)}$ reported an observational study of 376 patients with coronary artery disease in 2006. They found that patients with high ratio of absorbed cholesterol had higher cerebrovascular and cardiovascular events. In addition, the Myocardial Infarction Genetics Consortium Investigators in which Kathiresan was the principal investigator ${ }^{13)}$ identified the NPC1L1 gene exon sequence and detected NPC1L1 gene mutation carriers (nonsense mutation, splice mutation, and frameshift mutation) in 7364 coronary artery disease patients and 14,728 control subjects without coronary artery disease who were of European, African, and South Asian origin in 2014. They also performed genotyping in 22,590 coronary artery disease patients and 68,412 control subjects for specific gene mutations (p.Arg406X), and investigated the relationship between mutant gene expression, LDL level, and coronary artery disease risk in 2 groups. Fifteen NPC1L1 inactivated gene mutations were detected by sequencing, and the frequency of the heterozygous carrier for each mutation was approximately 1/650. They also found that the mean LDL value in the gene mutation carriers was $12 \mathrm{mg} / \mathrm{dL}$ lower than that in non-carriers $(\mathrm{p}=0.04)$, and the mutation carriers showed 53\% lower coronary artery disease risk, with statistical significance $(\mathrm{p}=0.008)$. However, the authors stated that further investigations were necessary to confirm their results to support the usefulness of cholesterol absorption inhibitors.

The previous reports suggest mixed results regarding the 

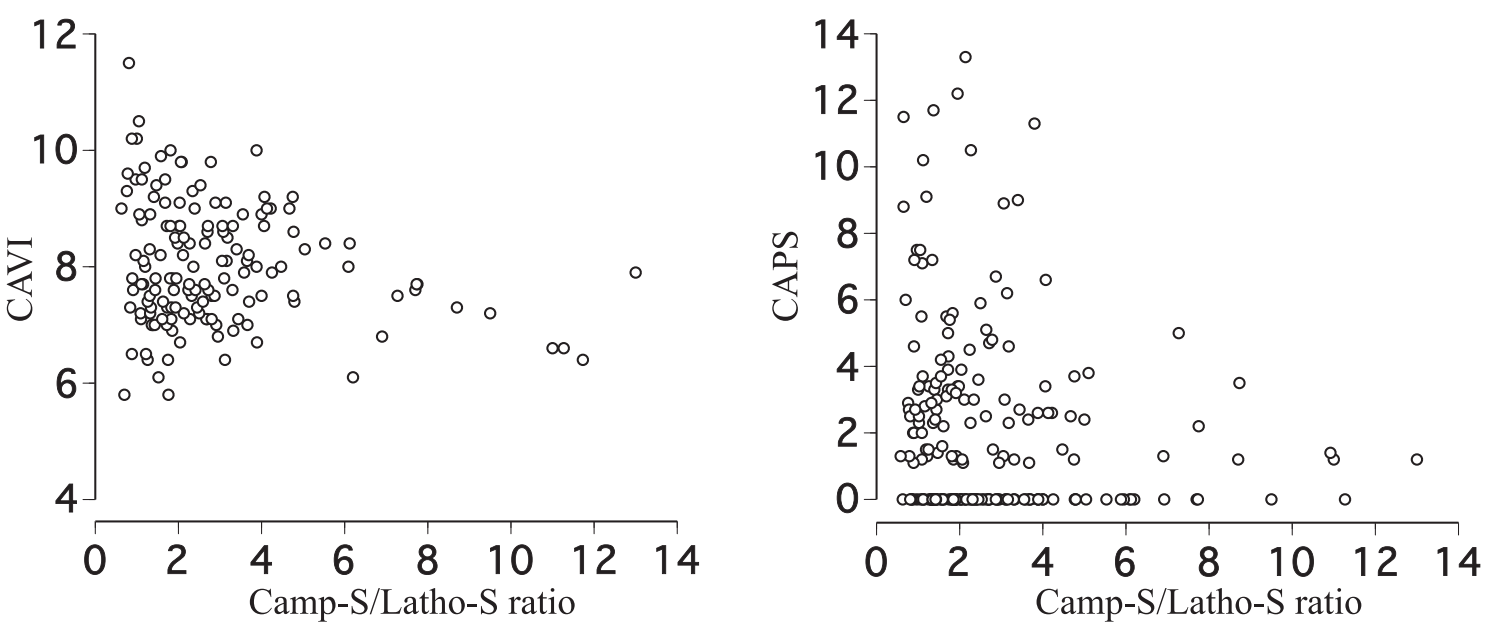

Figure 3. Relationship between CAVI (left panel) or CAPS (right panel) and absorbed/synthesized cholesterol balance (Camp-s/Lathol-s ratio). Both CAVI $(r=-0.20, \mathrm{p}=0.015)$ and CAPS $(r=-0.16, \mathrm{p}=0.023)$ showed weak but significant negative correlations to the absorbed/synthesized cholesterol balance.

Table 2. Univariate analysis of each risk factor for CAVI and CAPS

\begin{tabular}{lccccc}
\hline & \multicolumn{2}{c}{ CAVI } & & \multicolumn{2}{c}{ CAPS } \\
\cline { 2 - 3 } \cline { 5 - 6 } & $r$ & $p$ & & $r$ & $p$ \\
\hline Age & 0.58 & $<0.0001$ & & 0.33 & $<0.0001$ \\
BMI & 0.13 & 0.107 & & 0.09 & 0.197 \\
SBP & 0.26 & 0.001 & & 0.18 & 0.011 \\
DBP & 0.14 & 0.094 & & 0.16 & 0.019 \\
TG & 0.14 & 0.081 & & 0.11 & 0.121 \\
LDL/HDLratio & 0.15 & 0.068 & & 0.11 & 0.123 \\
FBS & 0.07 & 0.390 & & 0.11 & 0.057 \\
Lathosterol & 0.07 & 0.424 & & 0.12 & 0.096 \\
Campestrol & 0.24 & 0.002 & & 0.16 & 0.019 \\
Camp-S/Latho-S ratio & 0.20 & 0.015 & & 0.16 & 0.023 \\
\hline
\end{tabular}

Camp-S/Latho-S ratio: campestrol and lathosterol ratio, CAPS: carotid artery plaque score, CAVI: cardio-ankle vascular index

effect of absorbed cholesterol on atherosclerosis. Thus, studies that showed a favorable effect of absorbed cholesterol were conducted in healthy subjects, whereas studies that showed a negative effect were conducted in patients with atherosclerotic vascular diseases. Therefore, as shown in previous studies, the inhibition of both synthesis and absorption of cholesterol is effective in patients with atherosclerosis progression, and absorbed cholesterol may be effective to prevent atherosclerosis in subjects who has not yet experienced cardiovascular events. Our results support the hypothesis that absorbed cholesterol can prevent atherosclerosis in subjects without experience in cardiovascular events.

This study has several limitations. First, this was a crosssectional study, which did not assess the cardiovascular events of the participants. This limitation can be solved by a prospective observation of participating subjects, which may also provide us new insights. Second, in the present study, some study participants may have had various background risk factors of atherosclerosis, especially diabetes mellitus or impaired glucose tolerance. Increased cholesterol absorption
Table 3. Multivariate analysis of risk factors for CAVI and CAPS

\begin{tabular}{lrrrrr}
\hline & \multicolumn{2}{c}{ CAVI } & & \multicolumn{2}{c}{ CAPS } \\
\cline { 2 - 3 } \cline { 5 - 6 } & $\mathrm{F}$ & $p$ & & $\mathrm{~F}$ & $p$ \\
\hline Age & 75.57 & $<0.0001$ & & 25.70 & $<0.0001$ \\
SBP & 6.14 & 0.014 & & 2.17 & \\
DBP & 0.86 & & & 3.24 & \\
TG & 2.24 & & & & \\
LDL/HDLratio & 9.17 & 0.003 & & & \\
FBS & & & & 0.35 & \\
Lathostrol & & & & 1.21 & \\
Campesterol & 0.11 & & & 0.48 & \\
Camp-S/Latho-S ratio & 8.42 & 0.004 & & 6.12 & 0.014 \\
\hline
\end{tabular}

Camp-S/Latho-S ratio: campestrol and lathosterol ratio, CAPS: carotid artery plaque score

activity has been reported in diabetic patients ${ }^{14)}$. A previous large-scale clinical study of cholesterol absorption inhibitor showed that the cholesterol absorption inhibitor did not reduce the cerebrovascular and cardiovascular events in nondiabetic patients, whereas the events in diabetic patients were significantly reduced, suggesting that cholesterol absorption activity is influenced by patients' background factors. Thus, in diabetic patients, absorbed cholesterol may induce progression of atherosclerosis, resulting in different conclusions. Further detailed studies with larger numbers of subjects are necessary. Third, the quality of absorbed cholesterol, including oxidative (degraded) cholesterols, should be investigated. Oxidative cholesterols are produced by excess heat or storage in the air of meat and egg with enriched cholesterol. Retort foods, fried foods (potato chips, etc.), and other fast foods also contain oxidative cholesterols. One study reported a positive correlation between the number of fast-food stores and cerebral infarction events ${ }^{15)}$, which does not support our results that higher balance ratio of absorbed cholesterol can prevent atherosclerosis progression. To confirm our results, not only absorbed cholesterol but also oxi- 
dative cholesterol (oxysterol) should be measured. Fourth, the study participants did not take any medication. Recent studies have shown that the number of patients who received statin therapy has increased and that statin therapy upregulates cholesterol absorption. It is also important to investigate whether other non-stain lipid-modifying therapies have similar effects on cholesterol absorption. Lastly, we could not provide the mechanism of absorbed cholesterol balance effect on atherosclerosis prevention. In this study, balance of absorbed and synthesized cholesterols was significant predictive value for CAVI and CAPS, and CAVI significantly correlated with CAPS. These findings suggest the presence of mediators among these parameters. Further studies of atherosclerosis progression factors such as oxidative cholesterol intake (described above), inflammation markers, small dense LDL or oxidative LDL are necessary. We will continue to investigate to resolve these study limitations in the future.

We found in the present study that absorbed cholesterol is closely related to vascular elasticity and morphological changes in lipid metabolism, causing atherosclerosis. While in the current standard guideline of lifestyle, restriction of lipid intake is considered as the primary step to improve abnormal lipid metabolism, our results may provide new insights into nutritional guidelines for healthy subjects with pre-atherosclerotic condition.

Conflicts of Interest

There are no conflicts of interest

\section{References}

1. Simon A, Megnien JL, Levenson J. Coronary risk estimation and treatment of hypercholesterolemia. Circulation 1997; 96: 2449-52.

2. Cannon CP, Blazing MA, Giugliano RP, McCagg A, White JA, Theroux $\mathrm{P}$, et al. Ezetimibe added to statin therapy after acute coronary syndromes. N Engl J Med 2015; 372: 2387-97.

3. Japaridze L, Sadunishvili M. The short term effect of atorvastatin plus ezetimibe therapy versus atorvastatin monotherapy on clinical outcome in the acute coronary syndrome patients by gender. Kardiol Pol 2017; 75: 770-8

4. Matthan NR, Zhu L, Pencina M, D'Agostino RB, Schaefer EJ,
Lichtenstein AH. Sex-specific differences in the predictive value of cholesterol homeostasis markers and 10-year cardiovascular disease event rate in Framingham Offspring Study participants. J Am Heart Assoc 2013; 2: e005066.

5. Naslafkih A, Sestier F. Expected versus observed survival in 3 large population studies with HMG-CoA reductase inhibitors. J Insur Med 2000; 32: 155-62.

6. Davies JP, Levy B, Ioannou YA. Evidence for a Niemann-pick C (NPC) gene family: identification and characterization of NPC1L1. Genomics 2000; 65: 137-45.

7. Altmann SW, Davis HR Jr, Zhu LJ, Yao X, Hoos LM, Tetzloff G, et al. Niemann-Pick C1 Like 1 protein is critical for intestinal cholesterol absorption. Science 2004; 303: 1201-4.

8. Fassbender K, Lutjohann D, Dik MG, Bremmer M, Konig J, Walter $\mathrm{S}$, et al. Moderately elevated plant sterol levels are associated with reduced cardiovascular risk: the LASA study. Atherosclerosis 2008; 196: 283-8.

9. Gylling H, Halonen J, Lindholm H, Konttinen J, Simonen P, Nissinen MJ, et al. The effects of plant stanol ester consumption on arterial stiffness and endothelial function in adults: a randomised controlled clinical trial. BMC Cardiovasc Disord 2013; 13: 50.

10. Ras RT, Fuchs D, Koppenol WP, Garczarek U, Greyling A, Keicher C, et al. The effect of a low-fat spread with added plant sterols on vascular function markers: results of the Investigating Vascular Function Effects of Plant Sterols (INVEST) study. Am J Clin Nutr 2015; 101: 733-41.

11. Buchwald H, Varco RL, Matts JP, Long JM, Fitch LL, Campbell GS, et al. Effect of partial ileal bypass surgery on mortality and morbidity from coronary heart disease in patients with hypercholesterolemia. Report of the Program on the Surgical Control of the Hyperlipidemias (POSCH). N Engl J Med 1990; 323: 946-55.

12. Strandberg TE, Tilvis RS, Pitkala KH, Miettinen TA. Cholesterol and glucose metabolism and recurrent cardiovascular events among the elderly: a prospective study. J Am Coll Cardiol 2006; 48: 708-14.

13. Myocardial Infarction Genetics Consortium I. Inactivating mutations in NPC1L1 and protection from coronary heart disease. N Engl J Med 2014; 371: 2072-82.

14. Gylling H, Miettinen TA. Cholesterol absorption and lipoprotein metabolism in type II diabetes mellitus with and without coronary artery disease. Atherosclerosis 1996; 126: 325-32.

15. Morgenstern LB, Escobar JD, Sanchez BN, Hughes R, Zuniga BG, Garcia N, et al. Fast food and neighborhood stroke risk. Ann Neurol 2009; 66: 165-70. 